\title{
MANIFESTAÇÕES ORAIS DE USUÁRIOS DE DROGAS ILÍCITAS: UMA REVISÃO DE LITERATURA NARRATIVA
}

\author{
ORAL MANIFESTATIONS OF ILLICIT DRUG USERS: \\ A NARRATIVE LITERATURE REVIEW
}

Caio Felipe Dourado Melo'; Deonice Monteiro Camapum²; Emille Thayna Araujo e Araujo 3 ; Cyrene Piazera Silva Costa ${ }^{4}$

RESUMO: O abuso no consumo de drogas tem sido considerado um grave problema de saúde pública, já que os usuários mudam seu comportamento e se descuidam da saúde geral e bucal. Desta forma, o sistema estomatognático dos usuários de drogas apresenta diversas lesões causadas por essas substâncias químicas. Este estudo tem por objetivo demonstrar as manifestações orais consequentes do uso abusivo de drogas ilícitas por meio de uma revisão literatura narrativa, afim de mostrar a importância do cirurgião-dentista na equipe multidisciplinar de tratamento desses indivíduos. Ao término desta revisão foi possível evidenciar alterações bucais como: redução do fluxo salivar, cárie e doença periodontal.

PALAVRAS-CHAVE: Dependentes químicos. Saúde bucal. Alterações bucais em dependentes químicos.

\begin{abstract}
Drug abuse has been considered a serious public health problem, as users change their behavior and neglect general and oral health. In this way, the stomatognathic system of drug users presents diverse lesions caused by these chemical substances. This study aims to demonstrate the consequent oral manifestations of abusive use of illicit drugs through a narrative literature review, in order to show the importance of the dental surgeon in the multidisciplinary team of treatment of these individuals. At the end of this review it was possible to show that oral alterations such as: reduction of salivary flow, caries and periodontal disease are some of the main oral aspects present in illicit drug users.
\end{abstract}

KEYWORDS: Addicts. Oral health. Changes in oral addicts.

\footnotetext{
1 Discente do $2^{\circ}$ período curso de Odontologia e da Liga Acadêmica de Qualidade de Vida (LAQUAVI) da Universidade CEUMA. E-mail: caiodourado82@gmail.com

2 Discente do $2^{\circ}$ período curso de Odontologia e da Liga Acadêmica de Qualidade de Vida (LAQUAVI) da Universidade CEUMA. E-mail: dedecamapum@hotmail.com

3 Discente do $2^{\circ}$ período curso de Odontologia e da Liga Acadêmica de Qualidade de Vida (LAQUAVI) da Universidade CEUMA. E-mail: emilleemille.ara@outlook.com

4 Docente do curso de Odontologia e coordenadora da Liga Acadêmica de Qualidade de Vida (LAQUAVI) da Universidade CEUMA. E-mail: cyrenepiazera@ hotmail.com
} 


\section{INTRODUÇÃO}

Atualmente a dependência química é um fenômeno crescente em todo o mundo. Por isso é muito divulgado e discutido (PRATTA; SANTOS, 2009). Droga é qualquer substância natural e sintética que, administrada por qualquer via no organismo, afeta sua estrutura ou função (ALMEIDA et al., 2002). O uso abusivo de drogas, sejam lícitas ou ilícitas, produzem danos indesejáveis que repercutem na vida social, cultural, econômica e na saúde, tanto geral quanto bucal, de seus dependentes (DAVOGLIO et al., 2009).

As drogas ilícitas mais frequentemente relatadas por usuários no Brasil são maconha, cocaína, crack e heroína (PEDREIRA et al.; 1999). Todas são substâncias psicoativas, uma vez que modificam o estado de consciência do usuário. Os efeitos podem ir desde uma estimulação suave a alucinações. Essas substâncias interferem no funcionamento dos neurotransmissores, provocando alterações e distúrbios no comportamento do seu usuário (DÉA et al., 2004).

As principais alterações na cavidade bucal devido ao uso dessas substâncias, são xerostomia, experiência de cárie elevada, redução do fluxo salivar e capacidade tampão, bruxismo, perdas dentais, doença periodontal, halitose, quelite angular e estomatite (RIBEIRO et al., 2002; TOMITA et al., 2004; D’AMORE et al., 2011; GUPTA et al., 2012). Além disso, o uso dessas drogas pode causar mudanças comportamentais nos usuários, como por exemplo, mudança de humor e perda da autoestima (PEDREIRA et al., 1999; RIBEIRO et al., 2002). Desta forma, os dependentes químicos apresentam não só o sistema estomatognático mutilidado, como também o psicológico prejudicado (RIBEIRO et al., 2002).

Devido ao aumento do consumo dessas drogas no Brasil, a partir do século XX, o Ministério da Saúde estabeleceu o Programa Nacional de Atenção integrada ao usuário de álcool e outras drogas, tal iniciativa é voltada a criação de um serviço específico multidisciplinar para drogadependentes, considerando-se os diversos aspectos dessa problemática (FERREIRA; LUÍS, 2004). Antes a dependência química estava diretamente relacionada à assistência psiquiátrica (PRATTA; SANTOS, 2009).

A literatura odontológica apresenta uma escassa produção sobre o assunto. O conhecimento dos aspectos bucais do droga-dependente pelo cirurgião dentista pode evitar complicações durante o tratamento odontológico (PEDREIRA et al.; 1999; PEREIRA 2012; FALCÃO et al., 2015). Por conseguinte, a Odontologia, além de aliviar a dor, pode contribuir para a reabilitação psico-social dos droga-dependentes, auxiliando no desenvolvimento da autoestima e 
ampliando a sua interação social, já que a recuperação implica no resgate do ser humano em todos os aspectos, eliminando o significado psicológico da droga (RIBEIRO et al., 2002).

Desta forma, objetivo deste estudo foi demonstrar as manifestações orais consequentes do uso abusivo de drogas ilícitas, afim de mostrar a importância do cirurgião-dentista na equipe multidisciplinar de tratamento desses indivíduos.

\section{MATERIAIS E MÉTODOS}

Esta pesquisa foi uma revisão de literatura narrativa. O levantamento bibliográfico foi feito nos canais de busca Google acadêmico e PubMed utilizando os seguintes descritores: drogas ilícitas dependentes químicos e saúde bucal. A seleção do conteúdo foi baseada na conformidade dos limites dos assuntos aos objetivos da revisão.

\section{RESULTADOS E DISCUSSÃO}

\section{Saúde bucal e o usuário de drogas ilícitas}

A saúde bucal de um indivíduo depende do estilo de vida escolhida por ele. O uso abusivo de drogas ilícitas implica na saúde bucal desses usuários (PEREIRA et al., 2012). Os problemas frequentemente encontrados nos droga-dependentes por Shetty e Mooney (2010) foram baixa autoestima com a aparência dental, dentes quebrados pelo apertamento e erosão dental. Além disso, esses autores também encontraram maior perda dental nesses indivíduos que a população em geral, assim como uma higiene bucal insatisfatória.

Ainda, Ribeiro et al. (2002), Tomita et al. (2004), D’Amore et al. (2011) e Gupta et al. (2012) destacam a xerostomia, experiência de cárie elevado, diminuição do fluxo salivar e capacidade tampão, bruxismo, doença periodontal, quelite angular, estomatite e diminuição do limiar de dor como consequências do uso abusivo de drogas ilícitas para a saúde bucal.

Na Odontologia, o atendimento aos pacientes droga-dependentes é pouco estudado, apesar do crescente aumento desse fenômeno social (PEDREIRA et al., 1999; PEREIRA, 2012; FALCÃO et al., 2015). Para Shetty e Mooney (2010) existe uma desconexão entre os pesquisadores de saúde bucal e o uso abusivo de drogas ilícitas. Tomita et al. (2004) afirmam que muitos cirurgiõesdentistas desconhecem como atuar sobre essa parcela da população, embora o atendimento odontológico para grande parte desses indivíduos é uma resposta as suas necessidades, tanto estéticas e psicológicas quanto biológicas (PEREIRA et al., 2012). 


\section{Saúde bucal e o usuário de maconha}

A maconha tem os menos efeitos prejudiciais que o tabaco (COLODEL et al., 2009). Geralmente, os usuários dessa droga apresentam a condição bucal mais precária quando comparados aos não usuários (PEDREIRA et al., 1999).

O hábito de fumar maconha afeta a composição da microbiota bucal, que ocorre por causa da diminuição na tensão de oxigênio nas bolsas periodontais e pode levar a uma seleção de bactérias anaeróbicas. Também pode afetar a capacidade de resposta das células imunológicas e com isso alterar a genética celular para a susceptibilidade à doença periodontal (RIBEIRO et al., 2002; PEREIRA et al., 2012). Tal droga tem como princípio ativo TCH (tetra-hidrocanabinol), que interfere na produção das células de defesa, tornando o usuário suscetível à diversas complicações, como por exemplo, a candidíase, devido a imunossupressão (COLODEL et al., 2009; FALCÃO et al., 2015).

Além disso, a maconha pode afetar a capacidade tampão da saliva, propiciando a aparição de cáries; causa manchas no dente, alteração de paladar e olfato, halitose e retarda cicatrização póscirúrgica (FERNANDES et al., 2008; FALCÃO et al., 2015).

\section{Saúde bucal e o usuário de cocaína}

A cocaína pode estar associada à aparição de lesões bucais, e pode ser administrada de várias formas, como inalada, fumada e até esfregada na gengiva (REIS et al., 2002; LINS et al., 2010; CHAIBEN, 2011). O Tabela 1 abaixo, apresenta as principais lesões bucais segundo a forma de administração da cocaína (REIS et al., 2002; LINS et al., 2010; CHAIBEN, 2011).

Tabela 1 - Principais lesões segundo a forma de administração da cocaína

\begin{tabular}{ll}
\hline Forma de administração & Principais Lesões \\
\hline Fricção na gengiva & Recessões gengivais \\
& Erosão dental \\
& Perda óssea avançada \\
& Dor aguda na gengiva \\
& Xerostomia e/ou redução no fluxo salivar \\
\hline Inalação & Erosão do septo nasal \\
\hline Fumo & Queilite angular \\
& Ulceração \\
& Necrose na mucosa e gengiva \\
& Candidose pseudomembranosa \\
\hline
\end{tabular}


Entretanto, independe da forma de administração, a cocaína pode causar também hipertensão, taquicardia, infarto do miocárdio, acidente vascular cerebral, insuficiência renal aguda, convulsões, hemorragia pulmonar, insônia, ansiedade e alucinações. Devido a cocaína causar taquicardia e infarto do miocárdio, não se deve realizar tratamento odontológicos invasivos, para que se garanta a segurança na utilização dos vasoconstritores (CHAIBEN, 2011; BOTELHO; ROCHA; MELO, 2013; FALCÃO et al., 2015).

\section{Saúde bucal e o usuário de crack}

Nos últimos anos, o crack se espalhou por todo o país. Por ser uma forma mais barata da cocaína, todas as classes sociais e hoje mais de um milhão de brasileiros são dependentes dessa substância (MS, 2009).

Tal droga é obtida a partir da mistura da cocaína, água e hidróxido de sódio ou bicarbonato de sódio, sua forma é sólida, uma pedra, podendo ser fumada em latas, cachimbos e tubos (ANTONIAZZI et al., 2013). Ela é absorvida de forma rápida pela via de circulação pulmonar e causa imediata sensação de euforia e excitação, outros efeitos que podem aparecer devido ao uso dessa substância são: visão turva, tontura, vertigens, desorientação, paranoia, alucinações, agitação, comportamento agressivo, delírios, vômitos, tremores, insônia, dilatação das pupilas, hipertensão, hipertermia e taquicardia. Podendo produzir alterações na ativação neurofisiológica, no metabolismo e na circulação sanguínea (CHAIBEN, 2011; BOTELHO; ROCHA; MELO, 2013; FALCÃO et al., 2015).

Além desses prejuízos, o crack causa modificações nas condições bucais, já que os usuários têm maior ocorrência de cárie, erosão dentárias, doença periodontal, úlceras, candidíase oral e alterações nas células epiteliais. Estes problemas bucais ocorrem nos usuários de crack devido as alterações que o mesmo causa no sistema imune, associado ao seu contato local e a redução do fluxo salivar (NAPPO et al., 2001; ANTONIAZZI et al., 2013; FALCÃO et al., 2015).

\section{CONCLUSÃO}

As manifestações orais consequentes do uso abusivo de drogas ilícitas são alterações no fluxo salivar, cárie, doença periodontal e descaso com higienização bucal. Logo, uma vez que o uso abusivo de drogas ilícitas traz perturbações a homeostase oral, o cirurgião-dentista deve conhecer 
tais problemas bucais, assim poderá realizar uma melhor anamnese, exame clínico, e atendimento aos droga-dependentes.

\section{REFERÊNCIAS}

ALMEIDA, B.C.M.; ARAUJO, U.C.; SILVEIRA, F.M.; et al. A saúde bucal do dependente de drogas psicotrópicas. Pesq.Bras.Odontoped.Clin.Integr, v. 2, n.2/3, p. 120-126, 2002.

ANTONIAZZI, R.P., BORTOLOTTO, F.C.; BACKES, D.S.; ZANATTA, F.B.; FELDENS, C.A.; et al. Efeito do crack nas condições bucais: revisão de literatura. Braz. J. Periodontol., v. 23, n. $1,2013$.

BOTELHO, A.P.M.; MELO, V.H.; ROCHA, R.C. Uso e dependência de cocaína/crackna gestação, parto e puerpério. Femina, v. 41, n. 1, P. 23-32, 2013.

BRASIL Ministério da Saúde. O crack: como lidar com este grave problema. Portal da Saúde. 2009-12 disponível em:

http://portal.saude.gov.br/portal/saude/visualizar_texto.cfm?idtxt=33717\&janela=1

CHAIBEN, C.L. Avaliação da percepção do paladar em usuários crônicos de crack. Curitiba, PR. Originalmente apresentada como dissertação de Pós-graduação, Universidade Federal do Paraná, 2011.

COLODEL, E.V.; SILVA, E.L.F.M.; ZIELAK, J.C.; ZAITTER, W.; MICHEL-CROSATO, E.; PIZZATTO, E.; et al. Alterações bucais presentes em dependentes químicos. RSBO, v .6, n. 1, p. 44-48, 2009.

D'AMORE, M.M.; CHENG, D.M.; KRESSIN, N.R.; et al. Oral health of substance-dependent individuals: impact of specific substances. JSAT, v. 41, n. 2, p. 179-185, 2011. 
D’AVOGLIO, R.S.; AERTS, D.R.G.C.; ABEGG, C.; FREDDO, S.L.; MONTEIRO, L.; et al. Fatores associados a hábitos de saúde bucal e utilização de serviços odontológicos entre adolescentes. Cad. Saud. Pub., v .25, n. 3, p. 655-667, 2009.

DÉA, H.R.D.; DOS SANTOS, E.N.; ITAKURA, E.; et al. Inserção do Psicólogo de Prevenção ao Abuso de Álcool e Outras Drogas. Psicologia ciência e profissão, v. 24, n. 1, p. 108-115, 2004.

FALCÃO, C.A.M.; DOS SANTOS, R.O.; PEREIRA, R.M.S.; SILVA, T.S.O. et al. Saúde bucal em dependentes químicos. RICS, v. 2, n. 3, 2015.

FERNANDES, J.P.; BRANDÃO, V.S.G.; LIMA, A.A.S.; et al. Prevalência de lesões cancerizáveis bucais em indivíduos portadores de alcoolismo. Rev. Bras. Cancerol., v. 54, n. 3, p. 239-244, 2008.

FERREIRA, P.S.; LUIS, M.A.V. Percebendo as facilidades e dificuldades na implantação de serviços abertos em álcool e drogas. Enferm, v. 13, n. 2, p. 209-216, 2004.

GUPTA, T; SHAH, N; MATHUR, VP; et al. Oral health status of a group of illicit drug users in Delhi, India. Community dent. health, v. 29, n. 1, p. 49-54, 2012.

LINS, S.A.; GAETTI-JARDIM, C.; CIESIELSKI, F.I.N.; AGUIAR, R.C.M.S.; et al. Condições de saúde de pacientes de gênero feminino com dependência química. Salusvita, v. 29, n. 2, p.29-46, 2009.

NAPPO,S.A.; GALDUROZ, J.C.; RAYMUNDO, M.; et al. Changes in cocaine use as viewed by key informants: a qualitative study carried out in 1994 and 1999 in São Paulo, Brazil. J. Psychoactive Drugs, v. 33, n. 3, p. 241-53, 2001.

PEDREIRA, R.H.S.; REMENCIUS, L.; NAVARRO, M.F.L.; et al . Condição de saúde bucal de drogaditos em recuperação. Rev.Odontol.Univ.São Paulo, v. 13, n. 4, p. 395-399, 1999. 
PEREIRA, M.A.T. Uso de substâncias psicoativas e condições de saúde bucal de adolescentes em conflito com a lei. Londrina, PR. Originalmente apresentada como dissertação de Pósgraduação. Universidade Estadual de Londrina, 2012.

PRATTA, E.M.M.; SANTOS, M.A. O Processo Saúde-Doença e a Dependência Química: Interfaces e Evolução. Psic. Teor e Pesq, v. 25, n. 2, p. 203-211, 2009. RIBEIRO, E.D.P.; OLIVEIRA, J.A.; ZAMBOLIN, A.P.; LAURIS, J.R.P.; TOMITA, N.E.; et al. Abordagem integrada da saúde bucal de droga-dependentes em processo de recuperação. Pesq.Odontol.Bras., v. 16, n. 3, p. 239-245, 2002.

REIS, S.R.A.; SADIGURSKY, M., ANDRADE, M.G.S.; et al. Efeito genotóxico do etanol em células da mucosa bucal. Pesqui. Odontol. Bras., v. 16, n. 3, p. 221-5, 2002.

SHETTY, V.; MOONEY, L.J.; ZINGLER, C.M.; et al. The relationship between methamphetamine use and increased dental disease. JADA, v. 141, n. 3; p. 307-318, 2010.

TOMITA, N.E.; LAURIS, J.R. Abordagem integrada da saúde bucal de droga-dependentes em processo de recuperação. Pesq.Odontol.Bras., v. 16, n. 3, p. 239-245, 2004. 